\title{
In vitro Classical Conditioning of Abducens Nerve Discharge in Turtles
}

\author{
Joyce Keifer, Kim E. Armstrong, ${ }^{a}$ and James C. Houk \\ Department of Physiology, Northwestern University Medical School, Chicago, Illinois 60611
}

In vitro classical conditioning of abducens nerve activity was performed using an isolated turtle brainstem-cerebelIum preparation by direct stimulation of the cranial nerves. Using a delayed training procedure, the in vitro preparation was presented with paired stimuli consisting of a $1 \mathrm{sec}$ train stimulus applied to the auditory nerve (CS), which immediately preceded a single shock US applied to the trigeminal nerve. Conditioned and unconditioned responses were recorded in the ipsilateral abducens nerve. Acquisition exhibited a positive slope of conditioned responding in $60 \%$ of the preparations. Application of unpaired stimuli consisting of CS-alone, alternate $\mathrm{CS}$ and US, or backward conditioning failed to result in conditioning, or resulted in extinction of CRs. Latencies of CR onset were timed such that they occurred midway through the CS. Activity-dependent uptake of the dye sulforhodamine was used to examine the spatial distribution of neurons labeled during conditioning. These data showed label in the cerebellum and red nucleus during conditioning whereas these regions failed to label during unconditioned responses. Furthermore, the principal abducens nucleus labeled heavily during conditioning. These findings suggest the feasibility of examining classical conditioning in a vertebrate in vitro brainstem-cerebellum preparation. It is postulated that the abducens nerve CR represents a behavioral correlate of a blink-related eye movement. Multiple sites of conditioning are hypothesized, including the cerebellorubral circuitry and brainstem pathways that activate the principal abducens nucleus.

[Key words: classical conditioning, in vitro, abducens nerve, turtle, positive feedback, sulforhodamine]

Conditioned response learning is a simple form of associative learning. A promising model to study the neural mechanisms underlying associative learning is the classically conditioned eye-blink reflex (Thompson 1986; Glickstein and Yeo, 1990; Bloedel et al., 1991). In behaving mammals, this response consists of retraction of the globe into the orbit which allows the nictitating membrane/eyelid to sweep passively across the eye

\footnotetext{
Received Oct. 27, 1994; revised Feb. 17, 1995; accepted Feb. 21, 1995.

This study was supported by NSF Grant BNS-9109572 to J.K. J.C.H. was supported by PO1-NS-17489. We thank Dr. John Disterhoft for helpful comments on the manuscript.

Correspondence should be addressed to Dr. Joyce Keifer, Depart

ment of Anatomy and Structural Biology, University of South Dakota, School of Medicine, Vermillion, SD 57069

${ }^{a}$ Present address: Beckman Institute, University of Illinois, 405 North Mathews Avenue, Urbana, IL 61801

Copyright (C) 1995 Society for Neuroscience $\quad 0270-6474 / 95 / 155036-13 \$ 05.00 / 0$
}

in response to a corneal airpuff. When a previously neutral auditory conditioned stimulus is paired with the airpuff, following a number of paired stimulus presentations, the eyelid closes in response to the auditory stimulus. Previous studies have shown that the neural pathways important in conditioned and unconditioned blink responses are located in the brainstem and cerebellum. However, the cellular mechanisms underlying this form of motor learning remain elusive.

In vitro model systems of associative learning have been established in invertebrate species such as the marine mollusks Aplysia (Lukowiak and Sahley, 1981), Limax (Chang and Gelperin, 1980), Hermissenda (Farley and Alkon, 1987), and Pleurobranchaea (Mpitsos and Davis, 1973). These preparations have greatly facilitated our understanding of the neural mechanisms of learning and memory. Similar in vitro model systems of associative learning in vertebrates has previously been hampered by the technical limitations of maintaining the viability of large portions of intact neural tissue in isolation. This problem was overcome by the development of an in vitro brainstemcerebellum preparation from the turtle which takes advantage of this species' extraordinary resistance to anoxia (Keifer and Houk 1991). Extensive neural connections are preserved in this preparation which allow the activation of sensory pathways by direct electrical stimulation of the cranial nerves rather than by more natural stimuli such as an airpuff or tone.

Recently, an in vitro model of the turtle eye-blink reflex was developed (Keifer, 1993a). Neural discharge characteristic of the eyc-blink reflex was evoked by a single shock stimulus applied to the trigeminal nerve, which contains afferents from the face and eye, while recording from the ipsilateral abducens nerve, which contains efferent fibers that project to the eye muscles. This abducens nerve discharge was postulated to represent a neural correlate of the eye-blink reflex because it corresponded closely with extraocular EMGs recorded during eyeball retraction in a reduced preparation, and corresponded well with EMGs obtained during airpuff-evoked blinks in alert turtles. Hence, the abducens nerve response obtained in vitro appears to be a neural model of the behavioral eye-blink reflex.

Behaving turtles have previously been conditioned using a variety of classical and instrumental training paradigms (Andrews, 1915; Poliakov, 1930; Boycott and Guillery, 1962; Spigel, 1963; Pert and Bitterman, 1969; Graf and Tighe, 1971; Manton et al., 1972). A classically conditioned head withdrawal response to a tone was reported (Poliakov, 1930). Using a more complex discrimination paradigm, Andrews (1915) conditioned turtles to withdraw from a feeding station at the sound of a whistle and to approach at the sound of a bell. Brightness and olfactory discrimination learning has also been achieved (Boycott and Guillery, 1962; Spigel, 1963). 
In this study, the in vitro brainstem-cerebellum preparation was presented with paired stimuli consisting of a train stimulus applied to the posterior eighth nerve (CS), which contains primarily auditory fibers, and immediately preceded a single shock stimulus applied to the trigeminal nerve (US). The results show that this pairing procedure results in a classically conditioned abducens nerve response generated entirely in vitro. Application of unpaired stimuli fails to produce acquisition, or results in extinction of conditioned responses, suggesting that the close temporal association of the CS and US is required to generate the conditioned activity. This study represents a first step in establishing this in vitro preparation as a model system in which to examine associative learning in a vertebrate. Using this model, cellular and pharmacological studies of the neuronal mechanisms underlying this form of motor learning will compliment those studies in progress using whole animal preparations.

A preliminary report appeared in abstract form (Keifer and Houk, 1990).

\section{Materials and Methods}

In vitro turtle brainstem-cerebellum. Preparation of the in vitro brainstem-cerebellum has been described in detail elsewhere (Keifer and Houk 1991). Briefly, freshwater pond turtles (Chrysemys picta, $n=53$ ) of 4-6 inch carapace length were anesthetized by hypothermia $\left(0^{\circ} \mathrm{C}\right.$ Marcus, 1981). Following decapitation, the brain and cervical segments of the spinal cord were quickly dissected from the skull as the tissue was bathed in cold physiological saline. Care was taken to preserve intact cranial nerves of several millimeters in length. The preparation was transferred to the recording chamber where the telencephalon was transected at the level of the hypothalamus and discarded, and the dura covering the brainstem, cerebellum, and spinal cord were dissected away. The tissue was pinned to the bottom of the recording chamber ventral side up and the aqueduct of Sylvius was cannulated with the fire-polished tip of a glass pipette to allow perfusion of the brainstem through the fourth ventricle. The tissue was continuously bathed in physiological saline (2-4 ml/min) containing (in $\mathrm{mm}$ ) $100 \mathrm{NaCl}, 6 \mathrm{KCl}$, $40 \mathrm{NaHCO}_{3}, 2.6 \mathrm{CaCl}_{2}, 1.6 \mathrm{MgCl}_{2}$, and 20 glucose, which was constantly oxygenated with $95 \% \mathrm{O}_{2}, 5 \% \mathrm{CO}_{2}$ and maintained at room temperature $\left(22-24^{\circ} \mathrm{C}\right)$. When not in use, the preparation was transferred to a beaker containing physiological saline and placed in the refrigerator. In these conditions, the preparation was viable for at least $4 \mathrm{~d}$. Most preparations were surgically prepared $1 \mathrm{~d}$ prior to the experiment.

Stimulation and recording procedures. Smooth fire-polished glass suction electrodes were used for stimulation and recording of cranial nerves. The tip diameter of each electrode was designed to match the size of the nerve so that it fit snugly into the electrode. Neural activity characteristic of the in vitro eye-blink reflex was evoked by a single shock clcctrical stimulus $(0.1 \mathrm{mscc}$ duration, usually two times reflex threshold) applied to the trigeminal nerve (unconditioned stimulus, US), which contains afferents from the face and eye, while recording from the ipsilateral abducens nerve, which contains efferent fibers that normally project to the retractor bulbi, lateral rectus, and pyramidalis muscles of the eye and nictitating membrane (Underwood, 1970; BarbasHenry and Lohman, 1986, 1988; Barbas-Henry and Wouterlood, 1988). Some of the properties of the unconditioned eye-blink response recorded in the abducens nerve have been described previously (Keifer, 1993a). The conditioned stimulus (CS) was applied to the ipsilateral posterior root of the eighth nerve, which contains predominantly auditory fibers (Foster and Hall, 1978; Ten Donkelaar and Nieuwenhuys, 1979). Extracellular signals were amplified using a bandpass of $10 \mathrm{~Hz}$ to $3 \mathrm{kHz}$ and recorded on magnetic tape (Hewlett-Packard) and a chart recorder (Astro-Mcd).

Conditioning procedures. A delayed conditioning procedure, in which the CS precedes and overlaps with the US, was employed in the present study. In mammals, acquisition of conditioned responses to this training procedure is not dependent on structures rostral to the midbrain, particularly the hippocampus (Norman et al., 1977; Soloman et al., 1986; Mauk and Thompson, 1987; Moyer et al., 1990). The training protocol was designed with some modification after those used previously in conditioning studies on the alert rabbit. Paired CS-US conditioning stimuli consisted of a $100 \mathrm{~Hz}, 1 \mathrm{sec}$ train stimulus $(0.1 \mathrm{msec}$ pulses) applied to the posterior root of the eighth nerve (CS) which immediately preceded a single shock to the trigeminal nerve (US), and both stimuli terminated simultaneously. In experiments using the rabbit, CS-US intervals were typically between $250-500 \mathrm{msec}$ (Gormezano et al., 1962; McCormick and Thompson, 1984; Disterhoft et al., 1985. Yeo et al., 1985), although intervals ranging up to $1 \mathrm{sec}$ have proven adequate for conditioning (Schneiderman and Gormezano, 1964). Due to the cooler temperature of the turtle preparation $\left(22-24^{\circ} \mathrm{C}\right)$ and presumed slower conduction time as compared to the rabbit, a $1 \mathrm{sec}$ CSUS interval was chosen for the present study instead of a shorter CS train. A shorter CS-US interval has not yet been tested. Furthermore, the tone $\mathrm{CS}$ in behavioral experiments using mammals generally ranged between $800 \mathrm{~Hz}$ to $1 \mathrm{kHz}$, or consisted of white noise (Schneiderman and Gormezano, 1964; McCormick and Thompson, 1984; Yeo et al., 1985). Auditory nerve fibers of the turtle are most sensitive to stimuli hetween 100-500 $\mathrm{Hz}$ at room temperature (Patterson, 1966; Art and Fettiplace, 1984) and therefore a CS of $100 \mathrm{~Hz}$ was used. Intertrial intervals in the present study were $30 \mathrm{sec}$, the average interval typically used in bchavioral studies, and were not varied to reduce the complexity of the stimuli in this initial study. Fifty paired stimuli comprised a single pairing session and each session was followed by a 30 min rest period in which there was no stimulation. The rest period was introduced because studies using the rabbit model have varied widely in the number of pairing sessions (or blocks of paired stimuli) presented to the animal and these were given on a daily basis. Since it was not practical to apply blocks of paired stimuli on a daily basis in vitro, blocks were separated by a $30 \mathrm{~min}$ rest period in which there was no stimulation. Application of this stimulus protocol was continued until a conditioned response (CR) was acquired, which occurred most often by the second or third pairing session ( $71 \%$ of preparations showing acquisition). These parameters of the training protocol were therefore selected to customize the stimuli for our preparation.

Unpaired control stimuli were used to test for possible sensitization or pseudoconditioning effects. Control sessions consisted of presentations of the CS alone, alternate presentations of the CS and US such that the US followed the CS by $10 \mathrm{sec}$, and backward pairing in which the onset of the US immediately preceded the onset of the CS. Unpaired stimuli were applied either at the beginning of the experiment or followed paired stimuli to test for extinction of conditioned responses.

Amplitude of the conditioned stimulus. The intensity of the auditory nerve CS was selected in the following way. At high stimulus intensity, short latency neural discharge could be evoked in the abducens nerve. We have termed this the suprathreshold abducens nerve response. This stimulus also produced a behavioral eye-blink reflex and discharge recorded in the retractor bulbi muscle in a reduced preparation consisting of the brainstem-cerebellum in situ with intact peripheral connections to the eye (data not shown). Although speculative, one possibility is that this response represents a neural correlate of the acoustic startle reflex. The startle reflex is observed in behaving animals following a loud stimulus which results in a massive stereotyped flinch involving much of the body musculature including facial and neck muscles (Davis, 1984). If the CS was set ton low, conditioned responses failed to be acquired following numerous pairing sessions. Therefore, at the beginning of each experiment, the threshold for evoking any level of activity in the abducens nerve from auditory nerve stimulation was carefully determined. The CS was then set below this threshold value. Conditioned responses were obtained for a wide range of stimulus intensities. The CS was usually set between $77 \%$ and $50 \%$ of threshold levels ( $83 \%$ to $20 \%$ range; $65 \% \pm 17 \%$ mean \pm SD). Acquisition or failure to obtain acquisition did not depend solely on CS intensity as some preparations (32\%) that received CS amplitudes within this range failed to demonstrate acquisition. These data suggest that there are likely to be other unidentified factors in addition to the amplitude of the CS that affect acquisition in this preparation.

Data analysis. The conditioned response was defined as neural discharge recorded in the abducens nerve that occurred during the auditory nerve conditioned stimulus. Figure 1 shows an example of a $C R$ in response to a CS, followed by an unconditioned response to a US. Since the stimulus artifacts for the CS train were unidirectional (downward in Figs. 1, 2B, 3B), CRs were clearly visible as activity above baseline noise in the opposite (upward) direction. To be counted as a CR, the activity had to exceed a criterion level (dotted line in Hig. 1) conservatively set as $25 \%$ of the mean UR response. The latter was determined from 10 or more URs recorded before conditioning trials were begun. Amplitudes of URs and whether or not CRs exceeded the criterion were 


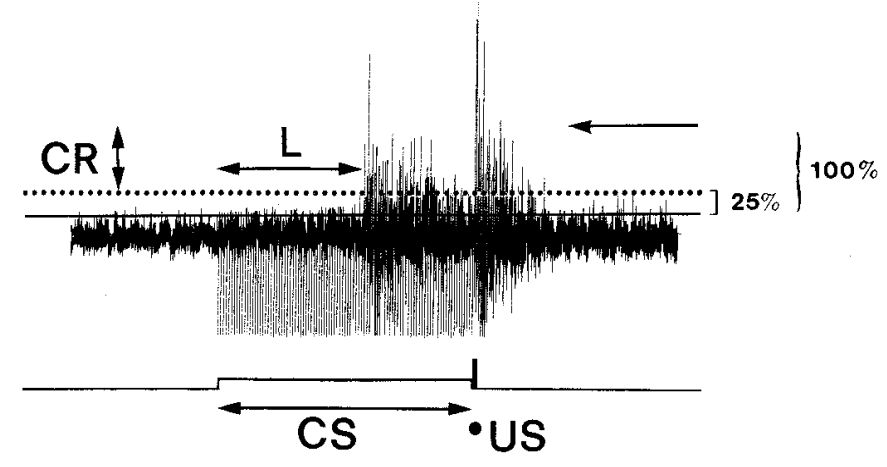

Figure 1. Illustration of the methods used to determine the presence and latency of conditioned responses $(C R)$. Top trace shows an extracellular recording of abducens nerve activity. Bottom trace shows the stimulus monitor. The top of the baseline nerve trace was lined up on the oscilloscope grid as illustrated by the solid line superimposed on the nerve recording. CRs were defined as abducens nerve activity that occurred during the $1 \mathrm{sec}$ conditioned stimulus $(C S)$ that were at least $25 \%$ of the mean amplitude of the unconditioned response. The arrow indicating $100 \%$ of the UR is a mean of 15 responses. The $25 \% \mathrm{CR}$ criterion is represented as a dotted line. In this example, a CR occurred midway through the CS and was followed by the UR. Latency $(L)$ of the CR was measured from the beginning of the CS to the point where the CR surpassed the $25 \%$ criterion level. The bottom trace shows the $1 \mathrm{sec}$ CS followed immediately by the single shock US.

estimated directly from the voltage envelopes recorded on a storage oscilloscope. To facilitate these measurements the baseline was aligned on one of the horizontal grids as illustrated by the solid line in Figure 1. In the illustrated trial, the US produced a brief stimulus artifact, and the UR was slightly larger than the mean UR (arrow) determined earlier in the experiment. Note that in estimating the amplitude of URs and $\mathrm{CRs}$, individual spikes that exceeded the vollage envelope were disregarded. Latency of CRs ( $\mathrm{L}$ in Fig. 1) was measured from the onset of the CS to the point where the neural discharge surpassed the criterion level. This criterion was chosen because it is not yet clear what level of neural discharge in the nerve results in eyeball retraction or nictitating membrane/eyelid movement, and because with this rather conservative threshold we believe that conditioned responses were more likely to be underestimated rather than overestimated. The number of conditioned responses reaching criterion in each session (for a total of 50 possible) were counted and the data were plotted in the form of acquisition curves. For greater resolution in the acquisition curve, the same data for each session were plotted as the number of CRs occurring in each block of 10 stimuli. In experiments in which backward pairing was used, CRs could not be determined during pairing due to the overlapping unconditioned response. In these experiments, the acquisition of CRs was assessed by using 10 test stimuli of CS-alone trials either immediately following the pairing session, or 2 test stimuli after each block of 10 paired stimuli for a total of 10 test stimuli. These data were plotted as percentage conditioned responses to the test stimuli.

Means \pm SEM or SD were calculated for grouped data. Statistical significance of overall differences between conditioned and unpaired procedures was determined by an analysis of variance (ANOVA) using sessions as a repeated measure. Individual comparisons for significance were made using two-tailed $t$ tests.

Labeling with sulforhodamine during abducens nerve CRs. To label neurons likely to participate in the abducens nerve conditioned responses, uptake of the activity-dependent fluorescent dye sulforhodamine 101 (Molecular Probes) was used (Keifer et al., 1992). Following the acquisition of CRs, a series of experinents was performed in which a final concentration of a $0.01 \%$ solution of sulforhodamine mixed with physiological saline was applied to the bath during the first two sessions of CS-alone extinction trials. In these conditions of sulforhodamine application during CS-alone stimuli, only CRs, but not URs, were elicited in the abducens nerve. These data for conditioning could then be compared to previously obtained information on the distribution of labeled neurons during unconditioned abducens nerve reflex responses (Keifer, 1993a) in order to identify brain regions that may be selectively laheled following acquisition. Typically, sulforhodamine was applied for a pe- riod of two consecutive CS-alone control sessions and the intervening 30 min rest period (for a total of $1.5 \mathrm{hr}$ ). During this time CRs were in the process of extinguishing but a number were recorded particularly during the first control session. The dye was removed from the bath and either control or pairing sessions without the dyc werc resumed to test the preparation for further extinction or reacquisition. Experiments were usually terminated after two to three sessions as a precaution to prevent dye from being washed out of labeled neurons during further testing. At the end of the experiment, the preparation was immersion fixed in $4 \%$ paraformaldehyde and processed histologically. Tissue was examined under rhodamine epifluorescence and labeled neurons were plotted using a drawing tube. Sections contained both brightly and faintly labeled cells, the faintly labeled cells being just barely visible above background. Photomicrographs of the quality of sulforhodamine labeling can be found in Figures 2 and 3 of Keifer et al. (1992) and in Figure 2 of Kjaerulff et al. (1994). Only the brightly labeled neurons were plotted as these were likely to be the more active cells.

\section{Results}

\section{Acquisition and extinction of in vitro conditioned abducens} nerve responses

The results are based on 53 brainstem-cerebellum preparations that received paired CS-US stimuli of which $32(60 \%)$ demonstrated acquisition to the conditioned stimulus. Examples of the acquisition and extinction of conditioned abducens nerve responses are shown for two different experiments in Figures 2 and 3. The number of CRs recorded in each session are plotted in panel $A$ of each figure, and the percentage of CRs for each session is plotted in blocks of 10 in panel $B$ for greater resolution than is shown in $A$. During the initial phases of pairing shown in Figure 2, no neural activity in the abducens nerve was evoked in response to the CS. An example of an extracellular recording from the abducens nerve is shown in Figure $2 B$ (section a). In this recording, the stimulus artifact produced by the CS is visible as downward deflections in the trace and the single shock evoking unconditioned reflex activity is visible as a large upward deflection. There was no response in the nerve to the CS shown in the example in section a. However, following about 100 paired stimulus presentations, acquisition was obtained in pairing session 3 (Fig. 2A,B). A nerve recording shown in Figure $2 B$ (section b) shows $C R$ activity that is approximately the same amplitude as the unconditioned response. In this experiment, the $\mathrm{CR}$ activity had an average latency of $319 \mathrm{msec}( \pm 171 \mathrm{SD}, n$ $=67$ ) with respect to the onset of the CS. The activity continued throughout the presentation of the CS until the US was initiated (Fig. 2B, b, d). Following the four pairing sessions, the preparation was presented with two sessions of unpaired CS-alone stimuli. Application of these stimuli resulted in gradual extinction of the conditioned response (section c). Two subsequent sessions of paired CS-US stimuli resulted in reacquisition of the conditioned response at a faster rate (section d).

Similar data were obtained in a different experiment shown in Figure 3. Gradual acquisition of conditioned responses was obtained during CS-US pairing. As can be seen in the recording shown in Figure $3 B$ (section $\mathrm{b}$ ), the conditioned responses in this case were large in amplitude and nearly all of them had an abrupt onset. This activity was sustained throughout the CS to the occurrence of the US. The latency of the CRs in this experiment averaged $369 \mathrm{msec}$ ( $\pm 160 \mathrm{SD}, n=55$ ). During control stimuli of CS-alone trials in sessions 5-6 extinction developed (c), and in this experiment minimal re-acquisition was obtained when the CS and US were once again paired for one session. Nine additional experiments in which the unpaired stimuli consisted of CS-alone trials resulted in response curves showing acquisition-extinction-reacquisition. These data are summarized 
A

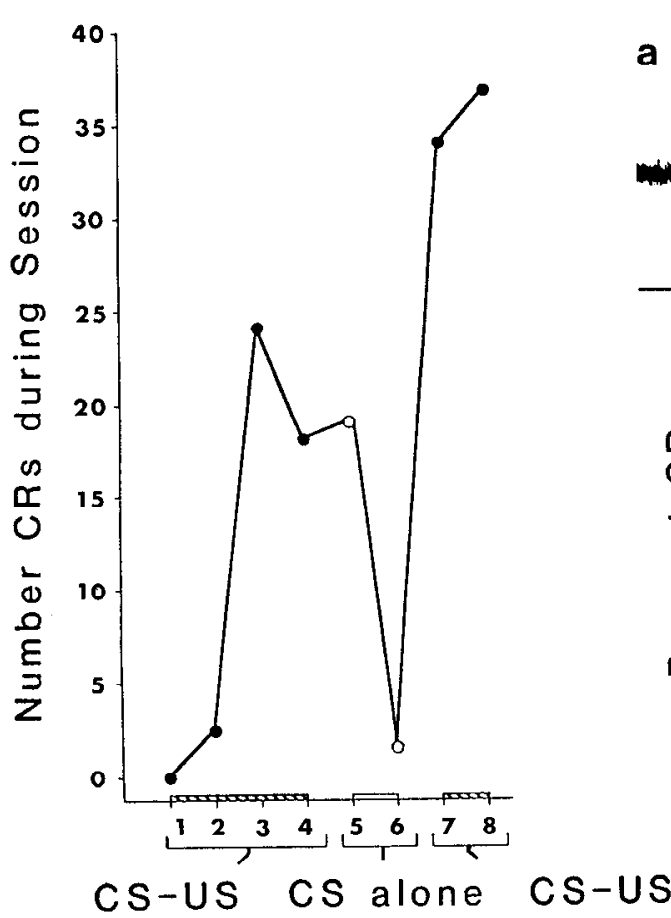

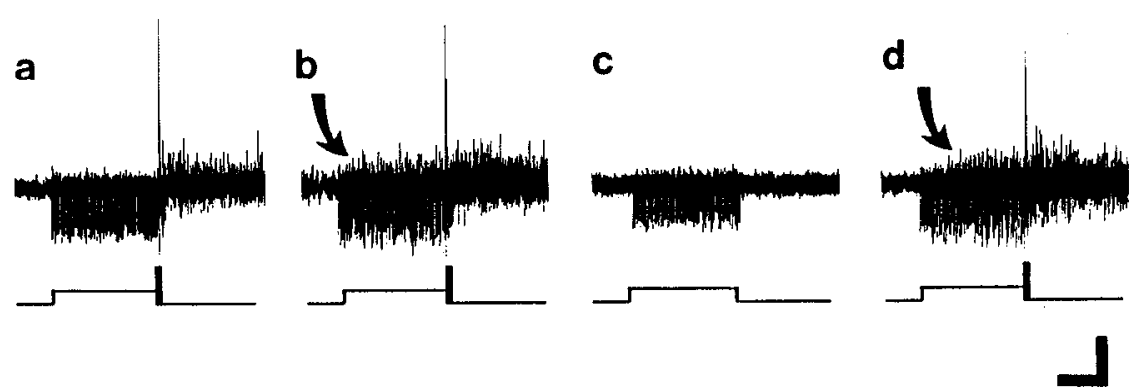

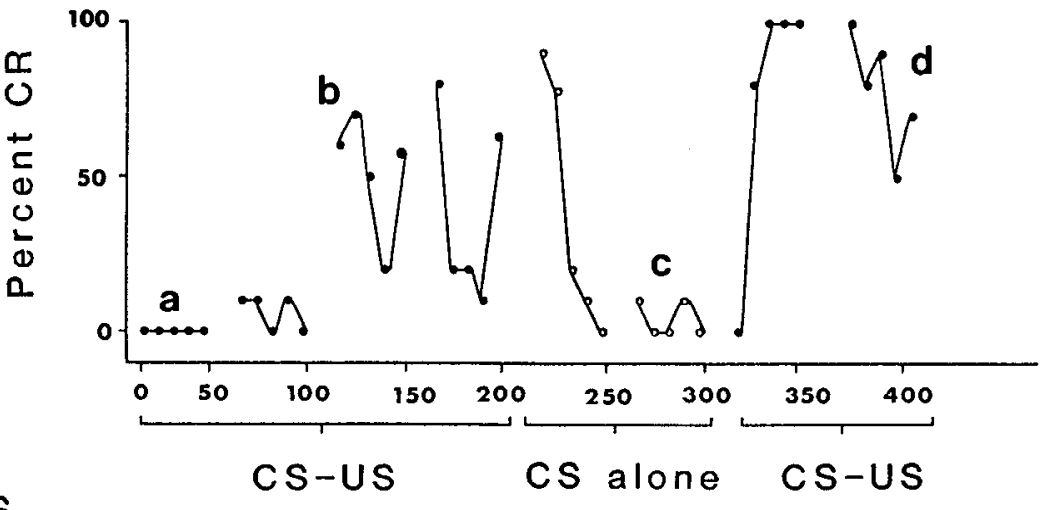

Figure 2. Acquisition and extinction of conditioned abducens nerve responses in vitro. A, Paired stimuli were applied to the auditory and trigeminal nerves during the first four training sessions $(C S-U S)$. The number of conditioned responses during each session is plotted. Following acquisition in sessions 1-4, unpaired CS alone control stimuli were applied in sessions 5-6, which resulted in extinction. Acquisition was obtained at a faster rate when paired CS-US stimuli were once again applied in sessions 7-8. B. Same data as in $A$, except that responses to the fifty stimuli applied per session are plotted as percentage CRs in blocks of 10 for greater resolution. Examples of abducens nerve recordings are shown for the periods during early pairing and before acquisition (a), during acquisition of conditioned responses (arrow, $b$ ), during extinction to unpaired stimuli (c), and during reacquisition $(d)$. The stimulus artifact associated with the CS is visible in the abducens nerve recording as downward deflections and immediately precedes a single shock US stimulus which is visible as a large upward spike. The CS and US stimulus monitors are shown below each nerve recording. Sulforhodamine was added to the bath in this case in session 5. Calibration: $50 \mu \mathrm{V}, 0.5 \mathrm{sec}$.

in Figure $4 A$ and are plotted as the percentage of CRs $( \pm$ SEM) recorded in each session. Beginning with CS-US pairing (solid bars), a positive slope of CR acquisition was demonstrated. The percentage of CRs increased at a rate of approximately $10 \%$ for each block of 50 paired stimuli. Maximal levels of conditioned responding have not been fully examined in these initial studies in order to test for abducens nerve responsiveness to unpaired stimuli in the same preparation. CS-alone stimuli (open bars) resulted in progressive extinction of CR responding. Reacquisition was obtained when CS-US pairing was resumed (solid bars).

Further evidence suggesting that the acquisition of conditioned responding is an associative event related to the close temporal pairing of the CS and US is shown in Figure $4 B$. Grouped data from two experiments are shown in which the preparation was initially presented with unpaired stimuli consisting of alternate CS and US (open bars). In these experiments, the number of CS and US stimuli applied to the preparation was exactly the same as those applied during the pairing sessions the only difference being that the stimuli were temporally separated by $10 \mathrm{sec}$. During the first five sessions, alternate CS and US stimuli failed to result in acquisition. In contrast, most preparations (71\%) demonstrating acquisition during pairing did so by sessions 2 and 3, and all other preparations showed acquisition by the fifth session. In session 6, the CS and US were paired (solid bars) which led to CR acquisition beginning in the second pairing session. A positive slope of conditioned responding was observed in subsequent pairing sessions. Presentation of unpaired stimuli at the beginning of the experiment slowed down acquisition (compare the $\sim 10 \%$ increase in CRs/session in Fig. $4 A$ with the $\sim 5 \%$ increase in Fig. $4 B$ ), a result which is typically found in behavioral studies (Gormezano et al., 1983). In two additional experiments, alternate CS and US stimuli were applied after acquisition to test for extinction. Data from one experiment are shown in Figure 5A. Acquisition of conditioned responses was obtained in pairing sessions 2 and 3. Presentation of alternate CS and US stimuli resulted in a reduction in the occurrence of CRs, and reacquisition was obtained when pairing of the CS and US was resumed. Thus, the separation of the CS and US by $10 \mathrm{sec}$ does not appear to support abducens nerve conditioned responding.

Another training paradigm to test for the effects of pseudoconditioning is backward conditioning. In this paradigm, the preparation is presented with stimuli in which the onset of the US immediately precedes the onset of the CS. Because there is no predictive value of the CS in signaling the US, learning in behaving animals is more difficult using this paradigm. In four experiments backward pairing failed to produce conditioned responses or resulted in the extinction of conditioned responses previously obtained during forward pairing. Data from one experiment are shown in Figure $5 B$ (solid line). Initially, the preparation was presented with backward paired stimuli for five ses- 


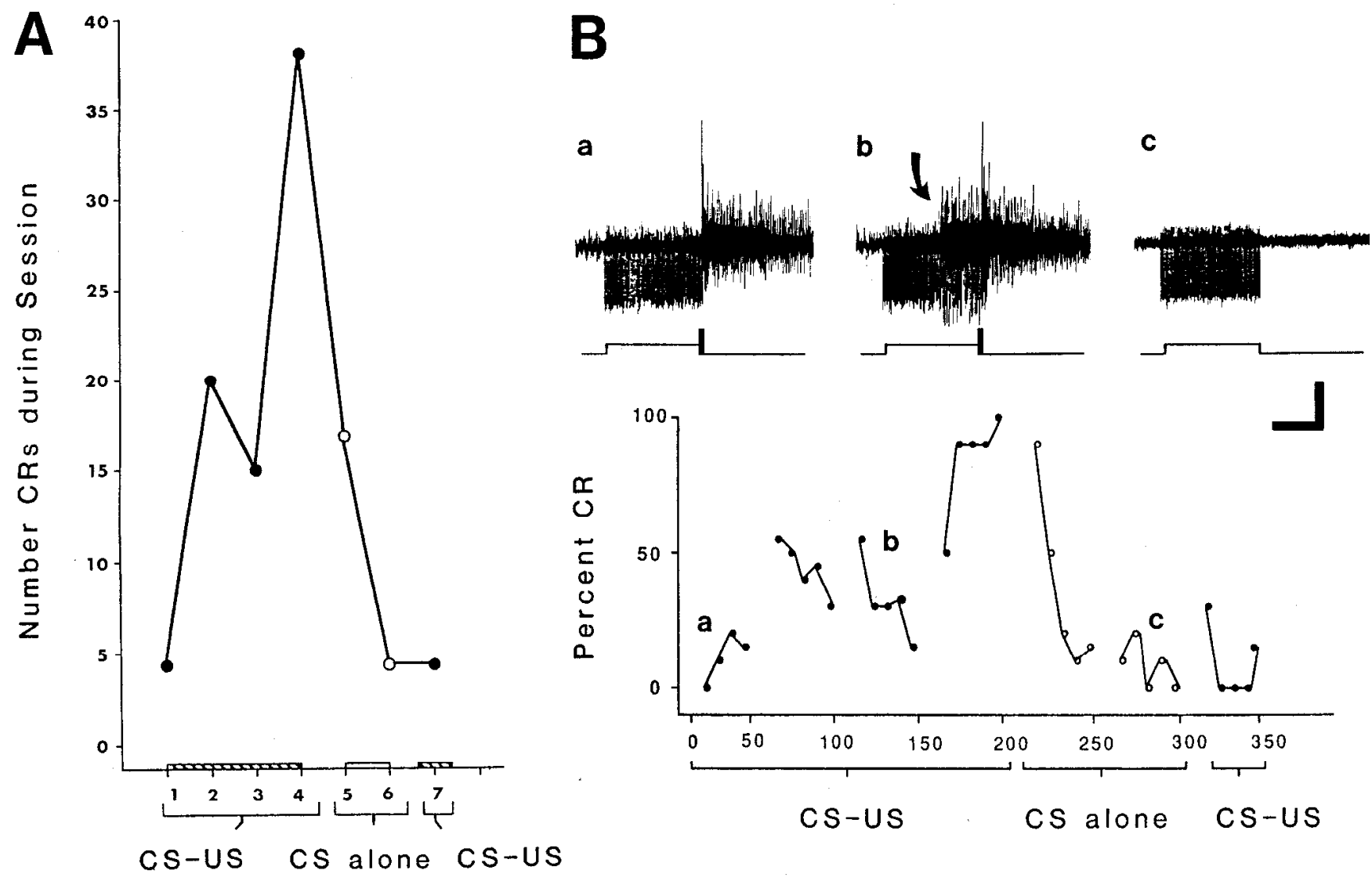

Figure 3. Acquisition and extinction of conditioned abducens nerve responses in a different experiment shows a pattern of responsiveness similar to the data shown in Figure 2. A, Number of CRs recorded in each session. Acquisition was obtained in sessions 1-4. Extinction occurrcd during presentation of the unpaired CS alone stimuli in sessions 5-6. Minimal reacquisition was obtained during the single pairing session 7 following the extinction trials. Sulforhodamine was applied to the bath in sessions 5 and $6 . B$, Same data as in $A$, except that responses are plotted as percentage CRs in blocks of 10. Examples of nerve recordings in $B$ show data prior to acquisition (a), during recording of CRs (arrow in $b$ ), and during extinction $(c)$. Calibration: $50 \mu \mathrm{V}, 0.5 \mathrm{sec}$.

sions (open dots), and no acquisition was observed. Presentation of forward paired stimuli in sessions 6-11 resulted in the acquisition of conditioned responses after about 150 stimuli beginning with session 9 (solid dots). These responses extinguished when backward pairing was resumed in sessions 12-14. Reacquisition was once again obtained with the application of forward paired stimuli in sessions 15-17. The mean number of CRs from three extinction experiments using backward pairing are plotted on the same graph (dashed line). These results clearly show that backward conditioning does not support the acquisition of conditioned responses. However, in one additional experiment that began with backward pairing, acquisition was observed in the second and third sessions. Following these stimuli, two sessions of alternate CS and US were applied, and these resulted in extinction. These stimuli were followed with three sessions of backward pairing and no acquisition was observed. Because the backward pairing procedure used in this study is comprised of CS and US stimuli that occur nearly simultaneously and are therefore closely paired, we are intrigued as to the potentially complex nature of the results using this training paradigm in vitro. Inconsistent effects of backward conditioning have been observed in the rabbit nictitating membrane preparation (Gormezano et al., 1983).

Grouped data comparing the percentage of conditioned responses oblained to paired and unpaired stimuli are shown in Figure $5 C$. The percentage of conditioned responses $( \pm S E M)$ is plotted for the last pairing session prior to control sessions of each experiment (paired), the last control session during extinction stimuli (unpaired), and the second pairing session following extinction trials (paired) for all experiments that received extinction trials between paired stimulus trials. Conditioned responding obtained during paired stimuli was significantly greater than responses obtained during unpaired extinction trials $(p<$ 0.01 ). Taken together, these data provide compelling evidence that acquisition of the abducens nerve conditioned response is due to the close temporal association of the CS and US and is not likely to be due to a nonassociative event such as sensiti zation or pseudoconditioning.

\section{Nature of abducens nerve CRs}

Upon inspection, the conditioned response recorded in the abducens nerve was clearly different from the unconditioned response. In a previous study, abducens nerve URs were found to have two distinct components: a short-duration synchronous discharge having a latency of 3-6 msec and a duration of 50-100 msec, followed by a long-duration component having a latency of 12-20 msec and a duration of several seconds (Keifer, 1993a). The CRs recorded in the present study were generally composed of smaller units and resembled the late component of the abducens nerve UR, as opposed to the large synchronous discharge of the early response. In addition, abducens nerve CRs also typically showed sustained activity that lasted until the occurrence 


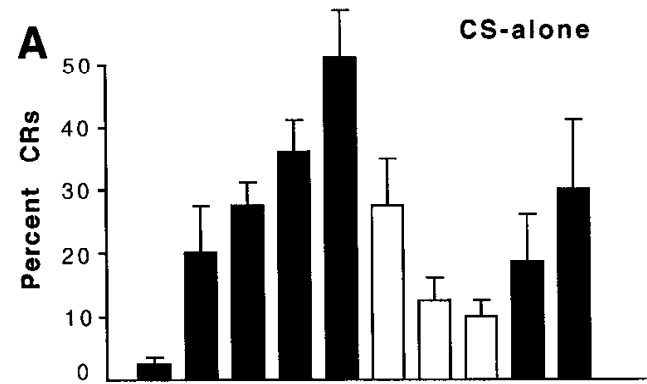

B

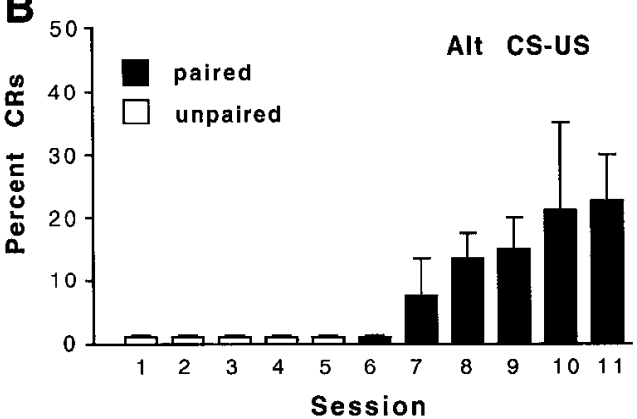

Figure 4. Grouped data summarizing results obtained from experiments using paired and unpaired CS-US stimuli. A, Summary of experiments in which CS-alone extinction trials were used $(n=10)$. Each bar represents the mean percentage CRs $( \pm S E M)$ in each training session. Solid bars, paired stimuli. Open bars, unpaired stimuli. Five sessions of paired CS-US stimuli resulted in a positive slope of CR acquisition. In sessions 6-8, unpaired CS-alone stimuli resulted in gradual extinction of conditioned responding. Paired stimuli in sessions 9-10 resulted in reacquisition. $B$. Summary of experiments that began with unpaired stimuli of alternate CS and US for five sessions $(n=2)$. No CRs were acquired to these stimuli in sessions 1-5. Paired CS-US stimuli were applied in sessions 6-11 and resulted in a positive slope of CR acquisition.

of the US. These observations are illustrated in an abducens nerve recording shown in Figure 6. Following acquisition, this preparation was presented with alternate CS-US stimuli. To the left of the trace in Figure 6 is a CS in which a CR was recorded with a latency of about $450 \mathrm{msec}$. For comparison, a UR was evoked several seconds later showing the short-latency, shortduration reflex component. Clearly, the $C R$ is not the same response as comprises the early component of the UR.

\section{Measurements of $C R$ latency}

Measurements of latency are an important definitional guideline for determining the presence of conditioned responses. In behaving animals, conditioned eye-blink responses typically have a variable latency with a minimum of 100-125 $\mathrm{msec}$ (Gormezano, 1972), as compared to the fixed-latency acoustic startle response which is 5-6 msec measured in the head or neck muscles (Cassella et al., 1986; Pilz et al., 1988). Figure 7A shows the mean latency $( \pm S D)$ of the onset of conditioned abducens nerve responses with respect to the onset of the CS for 11 preparations (cases 1-11), and the latency of the suprathreshold abducens nerve response for three preparations (cases 12-14). The conditioned responses were generally of a much longer latency and had a greater variability than the suprathreshold response. The latency of conditioned responses ranged from a mean of $191 \mathrm{msec}$ to $447 \mathrm{msec}$. Thus, CRs typically occurred midway through the CS interval. The minimum response latency observed during the CS was between 50 and $60 \mathrm{msec}$ with respect
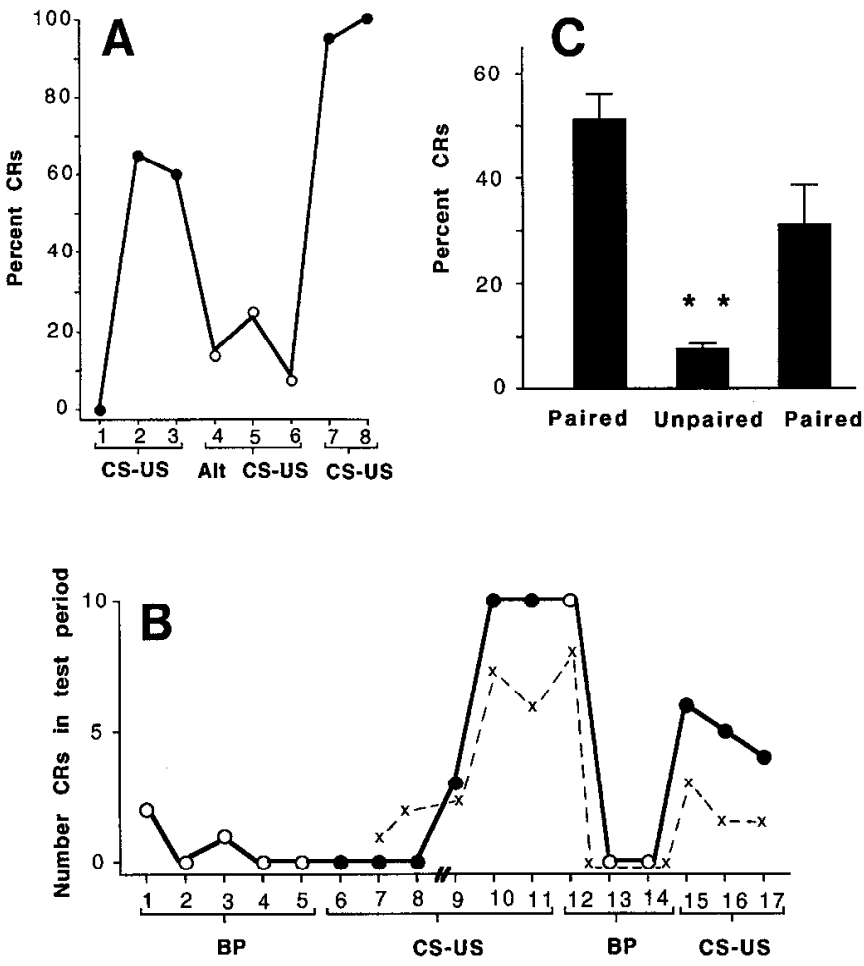

Figure 5. A, Data from one experiment in which the preparation was given extinction trials of alternate CS and US stimuli. Acquisition of CRs was obtained during the first three pairing sessions which were followed by unpaired stimuli in sessions 4-6. The alternate CS and US stimuli resulted in extinction of conditioned responding. Pairing was resumed in sessions $7-8$ and reacquisition was obtained at a faster rate than was observed during the initial pairing trials. Sulforhodamine was applied to the bath in sessions 5 and $6 . B$. The solid line shows data from one experiment in which acquisition of CRs was not supported by backward pairing $(B P)$ of the CS and US. In this experiment, acquisition of CRs was assessed by application of 10 test CS-alone stimuli at the end of each session. The experiment began with five sessions of backward paired stimuli during which time no acquisition was observed. Forward pairing began in session 6 which produced robust CR acquisition by session 10 (after 250 paired stimuli). These responses extinguished when backward pairing was once again presented in sessions 12-14. CRs were reacquired with subsequent pairing beginning in session 15. The crossbars between sessions 8 and 9 indicate that the preparation was left overnight in the bath. During this period, the electrodes remained in place, no stimulation was applied and the experiment was resumed the next day beginning with session 9 . The dotted line shows grouped data from three experiments using BP stimuli. Five sessions of forward pairing marked $7-11$ (sessions $1-5$ in these experiments) preceded three sessions of BP stimuli marked 12-14 which were followed by three sessions of forward pairing indicated by $15-17$. The means are indicated by $\times$. Acquisition to forward pairing was obtained, and presentation of BP stimuli resulted in extinction in the sessions marked 13-14. A small amount of reacquisition was observed following the BP stimuli. $C$, Summary of data comparing the percentage of conditioned responding to paired and unpaired stimuli from all experiments in which unpaired extinction trials were presented between paired stimulus trials $(n=17)$. Each bar represents the mean \pm SEM. Paired data (left har) are from the last pairing session prior to unpaired stimuli. Unpaired data are from the last control session (middle bar). Paired data (right bar) are from the second session after pairing was resumed. *, $p$ $<0.01$ significant difference between the unpaired group and either of the paired groups.

to the onset of the CS. In contrast, suprathreshold abducens nerve responses ranged from a mean of $4 \mathrm{msec}$ to $14 \mathrm{msec}$, with a minimum latency of 3-4 msec (significant difference between means of conditioned and suprathreshold reflex responses, $p<$ 


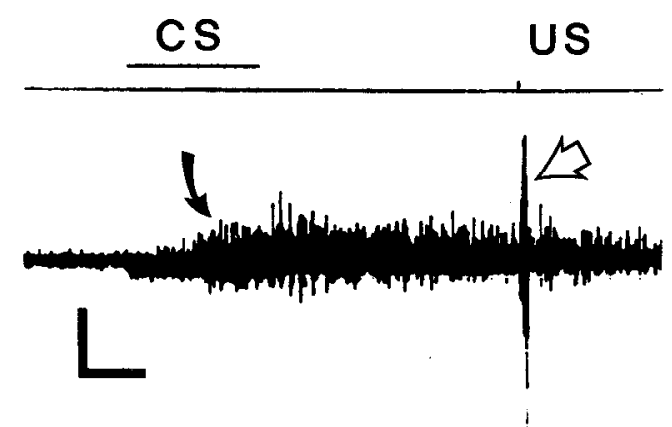

Figure 6. Example of an abducens nerve recording (bottom trace) comparing a typical CR and a UR. The stimulus monitor of the CS and US is shown in the top trace. A CR (to the left of the trace during a CS-alone stimulus, solid arrow) was comprised of small unit activity that demonstrated sustained discharge throughout the CS. In contrast, the UR (to the right, open arrow), which was evoked several seconds following the CS for comparison, consisted of a large synchronous discharge having a duration of $50-100 \mathrm{msec}$. This response is characteristic of the early phase of the UR. Calibration: $0.5 \mu \mathrm{V}, 0.5 \mathrm{sec}$.

$0.01)$. These data on latency support the conclusion that the conditioned responses recorded in vitro are not alpha, or reflex, responses.

\section{Habituation of the suprathreshold response}

Two important characteristics of the suprathreshold abducens nerve response distinguish it from conditioned responses. First, it has a significantly shorter latency than conditioned responses, as described above, and second, this response habituates. A 1 $\mathrm{sec}, 100 \mathrm{~Hz}$ train stimulus was applied to the posterior eighth nerve to evoke a suprathreshold response recorded in the ab- ducens nerve. As during conditioning, the stimuli were applied in blocks of 50 at an interval of $30 \mathrm{sec}$ followed by a $30 \mathrm{~min}$ period of no stimulation. Combined data from four experiments demonstrating habituation of the suprathreshold response are shown in Figure $7 B$. The mean number of abducens nerve responses $( \pm$ SEM) for each block of 10 stimuli in a session are plotted. As shown, the number of suprathreshold abducens nerve responses steadily decreases with the number of stimulus presentations, with some recovery at the beginning of each session. Thus, the suprathreshold abducens nerve response and the conditioned response have stimulus-response functions with opposite slopes. These data provide additional evidence that the CRs are not likely to be reflex responses.

\section{UR suppression}

An interesting phenomenon demonstrated by this in vitro preparation that has been observed in behaving mammals is that of conditioned suppression of the unconditioned response (Donegan, 1981). This is a diminution of the UR during paired stimulus presentations. Suppression of the UR during pairing was observed in 11 cases, all of which demonstrated acquisition. In all of these cases, the UR was completely absent for a number of paired stimuli in a session and then would reappear. Unlike adaptation, suppression occurred spontaneously and did not have a consistent relationship with the number of paired stimulus presentations. The first appearance of UR suppression could either precede or follow the first appearance of a conditioned response.

\section{Distribution of sulforhodamine-labeled neurons during abducens nerve $C R s$}

The fluorescent dye sulforhodamine has been previously shown to label cell bodies and dendrites in an activity-dependent man-
Figure 7. A, Mean latency of the onset of CRs wilh respect to the onset of the CS. The mean \pm SD is plotted for 11 experiments in which conditioning was acquired. Cases 12-14 show data of the mean latencies \pm SD of the suprathreshold abducens nerve response for comparison. Conditioned responses typically have longer $(p<0.01)$ and more variable response latencies as compared to the suprathreshold reflex. The number of observations for each case was, respectively, 37, 91, 59, 27, $55,67,97,44,20,32,70,27,50$, and 30. $B$, The suprathreshold abducens nerve response demonstrates habituation. The mean number of responses recorded in the abducens nerve in response to a strong train stimulus applied to the posterior eighth nerve are shown. Three sessions consisting of 50 stimuli each are plotted in blocks of 10 . Each data point is the mean \pm SEM from four preparations. The number of suprathreshold abducens nerve responses steadily declines with the number of stimulus presentations.
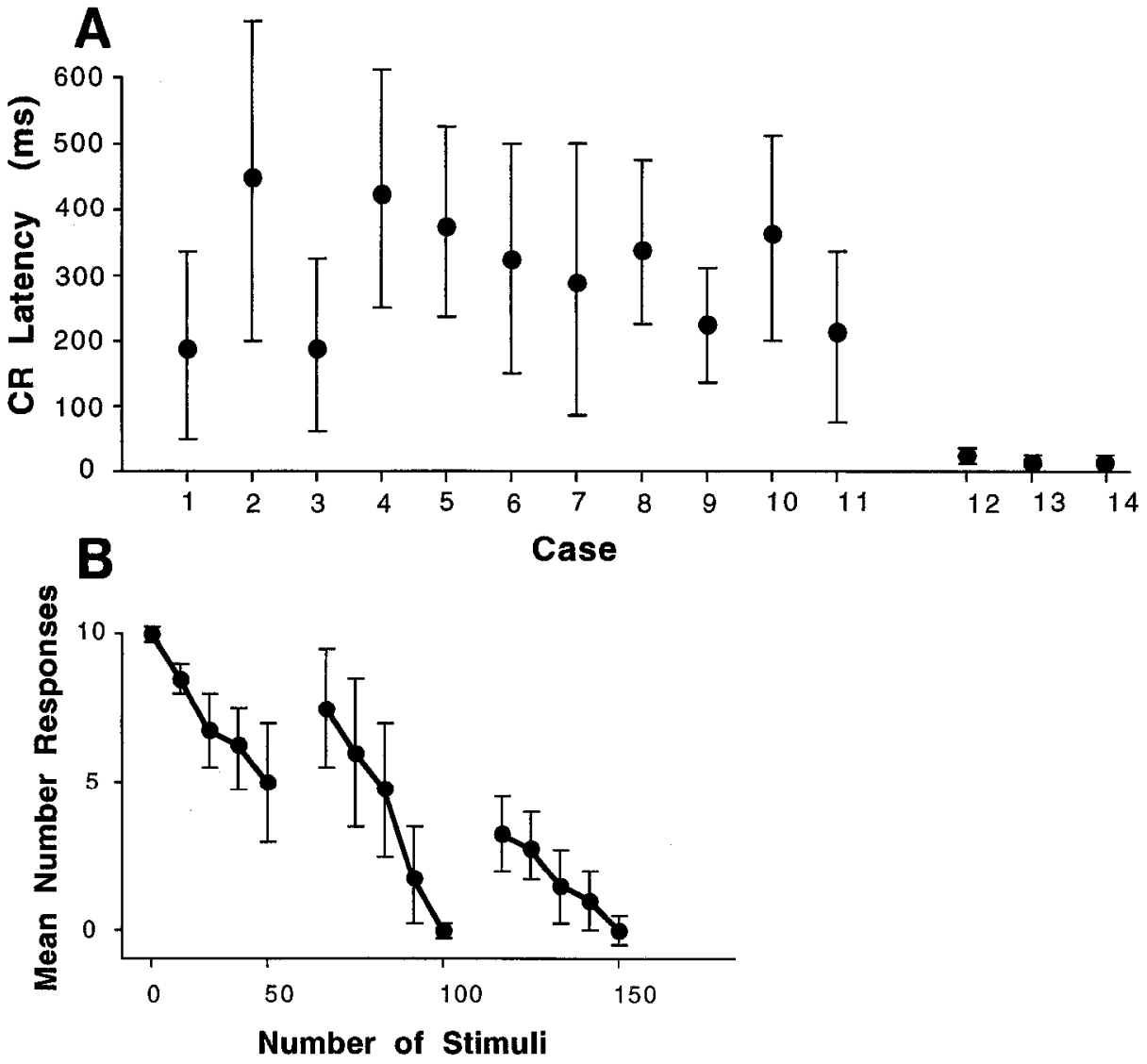
A
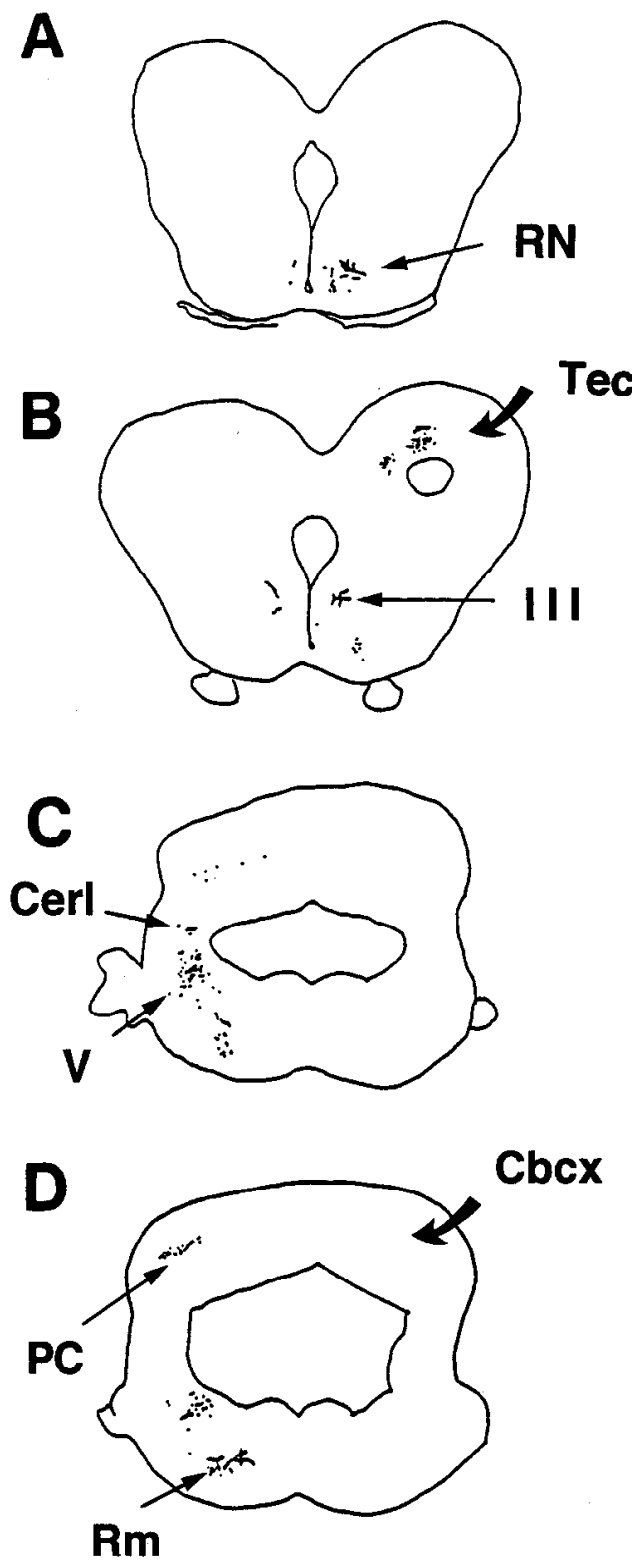

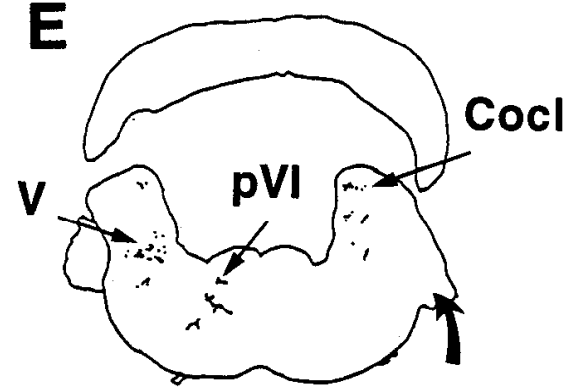

n VIII

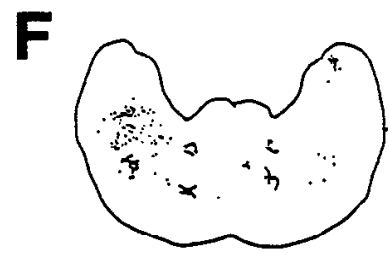

G

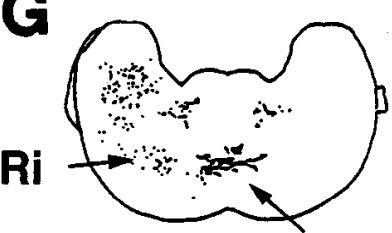

H

Rai

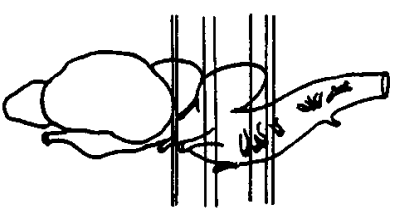

A

G $1 \mathrm{~mm}$
Figure 8. Example of the distribution of sulforhodamine label during recording of abducens nerve CRs in response to CS-alone stimuli. Labeled neuronal cell bodies and dendrites are plotted. Data are from the same experiment as shown in Figure 3 in which sulforhodamine was applied during sessions 5 and 6 . The CS was applied on the left side of the illustration. Labeled neurons were found in the contralateral red nucleus $(A, R N)$, the oculomotor nucleus bilaterally $(B, I I I)$, in several subdivisions of the rostral $(D, R m)$ and caudal $(F-G, R i$, ) reticular formation primarily ipsilaterally, in the inferior raphe nucleus $(G, R a i)$, the cochlear nucleus bilaterally $(E, C o c l)$, Purkinje cells ipsilaterally $(C-D, P C)$, the ipsilateral lateral cerebellar nucleus $(C, C e r)$, the ipsilateral trigeminal nucleus $(C-E, V)$, and the ipsilateral principal abducens nucleus $(E, p V I)$. The optic tectum $(T e c)$, cerebellar cortex $(C b c x)$, and nerve VIII ( $n$ VIII) are indicated. The relative rostrocaudal level of each illustrated section is shown in $H$. Scale bar applies to $A-G$. ner when the dye is applied to the bath and the preparation is electrically stimulated (Keifer et al., 1992; Keifer, 1993a). In the present study, activity-dependent uptake of sulforhodamine was used to label neurons in the brainstem and cerebellum that were active during the generation of abducens nerve conditioned responses. In these experiments, sulforhodamine was applied after acquisition and during the first two sessions of extinction trials consisting of CS-alone stimuli. Thus, during dye application only conditioned abducens nerve responses, but not unconditioned reflex responses, were elicited from the preparation. Ten preparations were tested with sulforhodamine during CS-alone trials, and of these 8 resulted in useful labeling. One case failed to label, and in another case the label was restricted to just a few neurons. In both of these experiments, three stimulus sessions followed the dye application resulting in a wash period of nearly $3 \mathrm{hr}$, whereas in the cases showing label dye application was followed by one or two sessions. Continuation of these two experiments may have contributed to the wash out of label. Three additional cases were tested with sulforhodamine in the same conditions as the above experiments, that is, stimulation of the posterior eighth nerve without the UR, except that these preparations failed to exhibit acquisition of CRs. The results of these cases showed that no label was present in the entire brainstem and cerebellum when the eighth nerve was stimulated but abducens nerve $\mathrm{CRs}$ were absent (see Fig. 9, hatched bars). One case in this group showed label in the ipsilateral cochlear nucleus.

The results of sulforhodamine labeling during CRs are best summarized by one typical case illustrated in Figure 8 . A series of transverse sections are shown indicating the distribution of labeled neurons for the same experiment illustrated in Figure 3. In this case, the dye was applied during extinction trials of CSalone stimuli in sessions 5 and 6 in which 21 conditioned responses were recorded. The experiment was terminated after session 7 as a precaution to prevent wash out of dye from stained neurons. Labeled neurons were found in the ipsilateral trigeminal nucleus $(\mathrm{V}, C-D)$, the principal abducens nucleus (p VI, $E$ ), areas of the reticular formation rostral $(\mathrm{Rm})$ and caudal $(\mathrm{Ri})$ to 


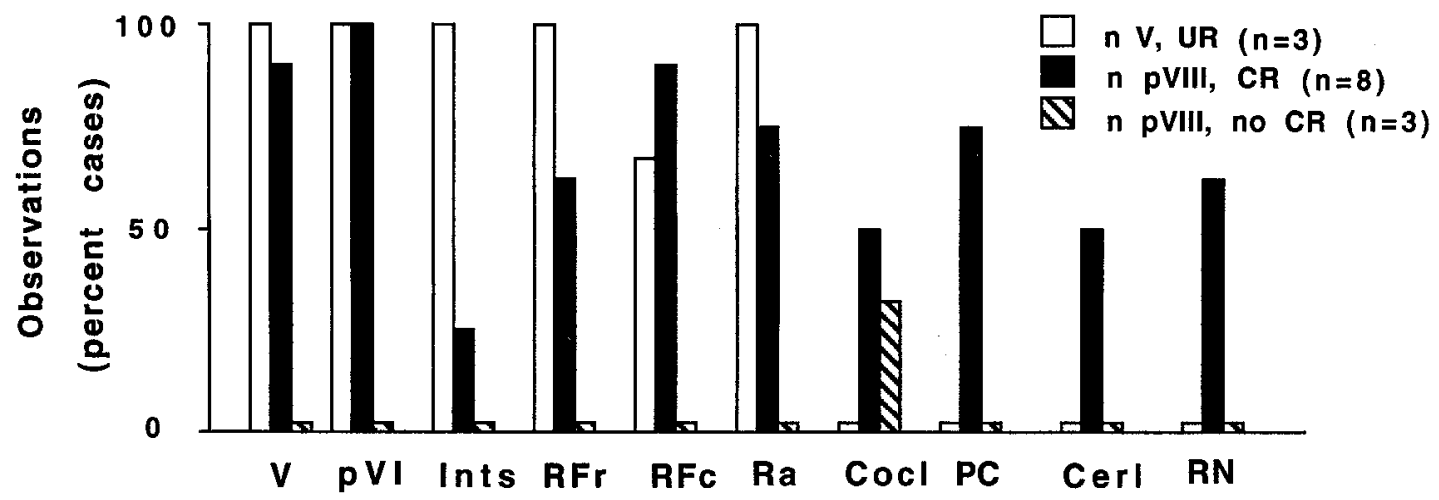

Figure 9. Summary of the spatial distribution of sulforhodamine label during stimulation of the trigeminal nerve while recording URs $(n=3$, open bars, data from Table 1, Keifer, 1993, cases 1-3; see also for 10 additional cases of sulforhodamine labeling during URs), during stimulation of the posterior eighth nerve while recording CRs $(n=8$, solid bars), and during stimulation of the posterior eighth nerve when no CRs were recorded ( $n=3$, hatched bars). The number of cases in which a specific nucleus was labeled is represented as the percentage of the total number of cases examined. Preparations tested with sulforhodamine following acquisition of CRs demonstrated more cases in which label was observed in the red nucleus $(R N)$, lateral cerebellar nucleus $(C e r l)$, Purkinje cells $(P C)$, and the cochlear nuclei $(C o c l)$ as compared to those cases in which sulforhodamine was applied during the UR. In contrast, the region of interneurons (Ints) ventrolateral to the principal abducens nucleus that consistently labeled during the UR, failed to label in most of the CR cases. Other abbreviations: $V$, trigeminal nucleus; $p V I$, principal abducens nucleus; $R F r$, reticular formation rostral to abducens nucleus; $R F c$, reticular formation caudal to abducens nucleus; $R a$, raphe nucleus. All labeled nuclei are ipsilateral to the site of stimulation except for PC and RF which are on either side, and RN which is contralateral. Ra is on the midline.

the level of the abducens nucleus $(D, F-G)$, and the inferior raphe nucleus (Rai, $G$ ). These sites were also found to label during the unconditioned reflex response (Fig. 9; see also Fig. 6 of Keifer, 1993a). Also similar to the unconditioned reflex cases, bilateral label in the oculomotor nucleus (III, Fig. $8 B$ ) was variable and was observed in $38 \%$ of the conditioned cases (three of eight cases labeled during the $\mathrm{CR}$ in the present study as compared to three of six cases during the UR reported in Keifer, 1993a). An area of tentatively identified interneurons involved in the unconditioned reflex pathway located ventrolateral to the principal abducens nucleus failed to label in the majority of conditioned cases. The accessory abducens nucleus failed to label with sulforhodamine under all experimental conditions tested. This result is not surprising in view of the fact that, as with other activity markers, there are many unknown steps between electrical stimulation and dye uptake.

Several nuclei were observed to label following conditioning that did not label with sulforhodamine during the unconditioned reflex response. These include the contralateral red nucleus $(\mathrm{RN}$, Fig. $8 A$ ), the ipsilateral cochlear nucleus (Cocl, $E$; labeled bilaterally in two cases), and the ipsilateral lateral cerebellar nucleus (Cerl, $C$ ). Purkinje cells (PC, $C-D$ ) failed to label during robust unconditioned responses, but did take up the dye ipsilaterally in most of the conditioned cases (bilaterally in four cases). Figure 9 directly compares the sulforhodamine-labeled nuclei that resulted during unconditioned reflex responses (open bars; data from Table 1 in Keifer, 1993a) with those regions that labeled following conditioning (solid bars). The percentage of cases from the total number tested in which a particular nucleus was observed to be labeled is plotted. Large differences in the patterns of labeling between the two sets of data are apparent. Following conditioning, the ipsilateral cochlear nucleus, Purkinje cells, ipsilateral cerebellar nucleus, and the contralateral red nucleus were observed to be labeled in $50-75 \%$ of the cases as compared to $0 \%$ of the unconditioned cases. In contrast, the group of interneurons that consistently labeled during the unconditioned reflex response failed to label in most cases of the conditioned group (there was sparse label in two cases). Labeled nuclei common to both groups were the ipsilateral trigeminal nucleus, the principal abducens nucleus, the rostral and caudal reticular formation and the raphe nucleus.

\section{Discussion}

The extraordinary ability of the turtle brain to survive for long periods of time in vitro raised the possibility that it might be possible to condition neural pathways by direct electrical stimulation of cranial nerves to mimic natural sensory input in the alert animal. In the present study, data has been presented suggesting the feasibility of examining classical conditioning in a vertebrate in vitro brainstem-cerebellum preparation. In the Discussion, we will evaluate the likelihood that conditioned abducens nerve responses are due to the close temporal association of the CS and US and are not due to a nonassociative event. Second, it will be hypothesized that the conditioned abducens nerve response represents a neural correlate of an eye-blink. Finally, the patterns of sulforhodamine label provide further evidence for conditioning, as well as insights into the functional basis of the conditioned pathway.

\section{Evidence for conditioning in vitro}

The in vitro turtle brainstem-cerebellum preparation exhibited a positive slope of conditioned responding recorded in the abducens nerve when presented with paired eighth nerve conditioned stimuli and trigeminal nerve unconditioned stimuli. Application of unpaired control procedures was used to assess the contribution of nonassociative processes to the likelihood of producing CRs. While the close temporal pairing of the CS and US supported conditioning, application of unpaired stimuli such as CS alone, alternate CS and US, and backward pairing clearly did not (Figs. 2-5). In the case of the alternate CS-US protocol, exactly the same number of stimuli were presented to the preparation as during conditioning except that the CS and US were separated by $10 \mathrm{sec}$ rather than overlapping. Future experiments will explore the limits of the temporal delay between CS and US that support CRs. A trace conditioning paradigm may require an intact hippocampus (ventro- and dorsomedial cortex) as it does in mammals (Solomon et al., 1986; Moyer et al., 1990). The results from the unpaired stimuli provide compelling evi- 
dence that $\mathrm{CR}$ acquisition is due to the close temporal association of the CS and US and is not due to a nonassociative process.

Measurements of latency are also an important guideline for determining the validity of conditioned responses. When a high intensity stimulus is applied to the auditory nerve, a short-latency discharge termed the suprathreshold abducens nerve response is recorded. In behaving mammals, conditioned eye-blink responses typically have a much longer latency (100-125 msec minimum) than the startle response (5-6 msec) and are more variable. Measurements of latency in the in vitro preparation show that CRs are significantly longer and have a greater variability as compared to the suprathreshold abducens nerve response (Fig. 7A). Moreover, CRs occurred midway through the CS interval, an important timing feature of conditioned responding found in mammals (Perrett et al., 1993). These latency data suggest that the CRs recorded in vitro are not alphid, or reflex, responses.

The CRs recorded in vitro are, moreover, not likely to be reflex responses because the latter habituate. Activation of the suprathreshold abducens nerve reflex results in progressively smaller responses (Fig. $7 B$ ). This is also true of the mammalian startle response (Prosser and Hunter, 1936; Leaton and Supple, 1986). Thus, while CRs demonstrate a positive slope of acquisition, suprathreshold responses have a negative slope.

These data strongly support the conclusion that it is possible to classically condition a vertebrate reflex pathway entirely in vitro. While the results obtained in this reduced preparation may not reflect the complexity of alert animal preparations, the requirement for paired stimuli and the long latencies of the CRs show promise for this preparation in cellular studies of conditioning.

\section{Behavioral correlate of the in vitro abducens nerve $C R$}

Among the many types of eye movements, the abducens nerve mediates blink-related and vestibular-induced eye movements. In most mammals, eye retraction is associated with movement of the nictitating membrane/eyelid, or eye-blink, which is generated by cocontraction of nearly all the extraocular muscles including abducens nerve mediated contraction of the retractor bulbi and lateral rectus muscles (Evinger and Manning, 1993). The same is true of the turtle, except that additionally movement of the nictitating membrane is generated by active contraction of the pyramidalis muscle. This muscle is also innervated by the abducens nerve. The pattern of muscle activation during eye movements is different from the pattern during blinks. For example, abduction of the eye involves contraction of the lateral rectus muscle accompanied by no activity in the retractor bulbi muscle. At present, the behavioral correlate of the in vitro abducens nerve conditioned response is not known. This difficulty arises because the posterior eighth nerve contains vestibular fibers in addition to a predominance of auditory axons. However, we believe it more likely that the CRs correspond to blink-related behavior rather than to vestibular-induced eye movements for the following reasons. First, stimulation of the posterior eighth nerve in a reduced preparation with intact peripheral attachments to the eye resulted in a behavioral eye-blink and discharge recorded in the retractor bulbi muscle (unpublished data). The retractor bulbi muscle is not active during eye movements (Delgado-Garcia et al., 1990). Second, we have been unsuccessful in generating horizontal eye movements in vitro from electrical stimulation of the posterior eighth nerve. Blink-related eye retraction can be readily evoked by stimulation of the tri- geminal (Keifer, 1993a) or eighth nerve. Third, the application of sulforhodamine during CRs failed to label the vestibular nuclei as would be expected during vestibular-induced eye movements. These nuclei are well labeled with sulforhodamine following electrical stimulation of the anterior eighth nerve, which contains axons of the vestibular apparatus (L. J. Larson-Prior, personal communication).

\section{Sulforhodamine-labeled pathways}

Sulforhodamine 101 is internalized by active neurons when the in vitro brainstem-cerebellum is bathed in the dye and electrically stimulated. Sulforhodamine was used previously to visualize the spatial distribution of motor-related activity in the cerebellorubral circuit (Keifer et al., 1992), and to label pathways active during the unconditioned in vitro eye-blink reflex (Keifer, 1993a). Sulforhodanine labeling appears to be dependent on synaptic activity. Evidence in favor of this interpretation comes from studies in which sulforhodamine staining was correlated with the presence of burst discharge recorded extracellularly from the red nucleus (Keifer et al., 1992). Blockade of the bursts with excitatory amino acid receptor antagonists resulted in failure of dye uptake in the red nucleus. In a different study, sulforhodamine was used to label pathways active during stimulation of the trigeminal nerve (Keifer, 1993a). Preparations showing strong abducens nerve activity demonstrated dye uptake in the trigeminal and principal abducens nuclei, in presumed interneurons, and in regions of the reticular formation. The patterns of uptake were dramatically altered with addition of excitatory amino acid receptor antagonists. Together, these studies provide strong evidence that sulforhodamine uptake is activity dependent. However, the issue of sensitivity of our staining protocol must be addressed. Keifer (1993a) noted that motoneurons of the accessory abducens nucleus, known to be active during abducens nerve reflexes, failed to label in all cases. Variability of label in our earlier study was also observed (Keifer et al., 1992). Using a different staining procedure, patterns of activity-dependent label were reported in the isolated neonatal rat spinal cord following chemically induced locomotion (Kjaerulff et al., 1994). Nonlocomoting preparations lacked staining. However, these authors reported variable labeling of motoneuron pools. It may be that some motoneurons are more resistant to sulforhodamine staining than others for as yet unexplained reasons. Similar variability in patterns of labeling have been observed with other activity markers such as ${ }^{14} \mathrm{C}$-2-deoxyglucose and c-fos (Sharp et al., 1989). While sulforhodamine labeling provides valuable and provocative insights into brain regions that might be active, it is important not to overinterpret these data but to let them guide further experiments.

In the present study, the spatial distribution of sulforhodamine label during conditioned ahducens nerve responses was examined. These data on conditioning can be compared to previously obtained data on the distribution of label during the unconditioned abducens nerve response (Keifer, 1993a). Two intriguing results were found with sulforhodamine. First, the distribution of labeled neurons suggests that there is a greater involvement of the cerebellorubral circuitry following conditioning than is observed during the unconditioned response. Labeled regions included the ipsilateral cochlear nucleus, Purkinje cells (although these did label during weak reflexes; Keifer, 1993a), neurons located laterally in the ipsilateral medullary reticular formation that may comprise in part the lateral reticular nucleus, the ipsilateral lateral cerebellar nucleus and the contralateral red nucle- 
us. These results contrast with those obtained during URs in which the cerebellorubral circuitry failed to label. Furthermore, patterns of labeling obtained during CRs contrast with the complete lack of staining when no CRs were recorded. These results are consistent with theories of the neural control of eye-blink conditioning that suggest an essential involvement of the cerebellum and red nucleus (Thompson, 1986; Glickstein and Yeo, 1990; Desmond and Moore, 1991).

A second finding from the distribution of sulforhodamine is that while the principal abducens nucleus labeled heavily, an area of interneurons ventrolateral to the principal nucleus failed to label during conditioning. This region contains significant label during the unconditioned reflex. The implications of these latter results lead to an hypothesis of the origin of the conditioned abducens nerve response and is discussed below.

\section{Interpretation of activated pathways and their role in conditioning}

Figure 10 summarizes our interpretation of the UR and CR pathways in the in vitro turtle preparation. As shown previously (Keifer, 1993a), the abducens nerve UR has two physiologically distinct components and is mediated by partially separate circuits, illustrated in Figure 10A. The early component is postulated to be produced by direct and interneuronal (Ints) pathways from the trigeminal nucleus (V) to the accessory abducens nucleus (acc VI). In contrast, the late component utilizes regions in the reticular formation (RF) in addition to nucleus $V$ and sends its output through the principal abducens nucleus (p VI). Short and long latency pathways mediating the mammalian eyeblink reflex have also been postulated (Keifer, 1993a, for references).

Areas of sulforhodamine labeling common to CRs and URs suggest that CRs recorded in the abducens nerve are generated by a final stage of output from the late UR circuit and typically do not involve the early UR circuit, a finding that is consistent with previous studies in mammals (Cegavske et al., 1979; Disterhoft et al., 1985; Delgado-Garcia et al., 1990; Steinmetz et al., 1992). Correspondingly, the CR pathway illustrated in Figure $10 B$ includes those portions of Figure $10 A$ that generate the late UR, namely nucleus $\mathrm{V}$ and the reticuloabducens network. This network is represented in the lower box in Figure $10 B$. In addition, the CR pathway includes the cerebellorubral circuitry, shown in the upper box of Figure $10 B$, which was labeled with sulforhodamine. The cerebellorubral network interconnects the red nucleus (RN), lateral reticular nucleus (LRN), and the lateral cerebellar nucleus (Cerl; mammals: reviewed in Houk et al., 1993; Keifer and Houk, 1994; turtles: Sarrafizadeh and Houk, 1994). Cerl is the reptilian equivalent of the mammalian anterior interpositus nucleus which has been implicated in conditioned responding (McCormick and Thompson, 1984; Thompson, 1986; Steinmetz et al., 1992).

Single-unit recording data from mammals reveal neuronal populations likely to participate in the CR output pathway. Purkinje cell activity in cerebellar hemispheral lobule VI which projects to nucleus interpositus preceded the initiation of CRs by as much as 100-200 msec (Berthier and Moore, 1986). Berthier and Moore (1990) recorded neuronal activity that preceded the $\mathrm{CR}$ by about $60 \mathrm{msec}$ in the rabbit nucleus interpositus anterior. In the red nucleus, CR-related activity preceded the response by about 20-40 msec (Desmond and Moore, 1991). Thus, these lead times suggest a flow of information from the cerebellar Purkinje cells to the anterior interpositus to red nucleus. Other
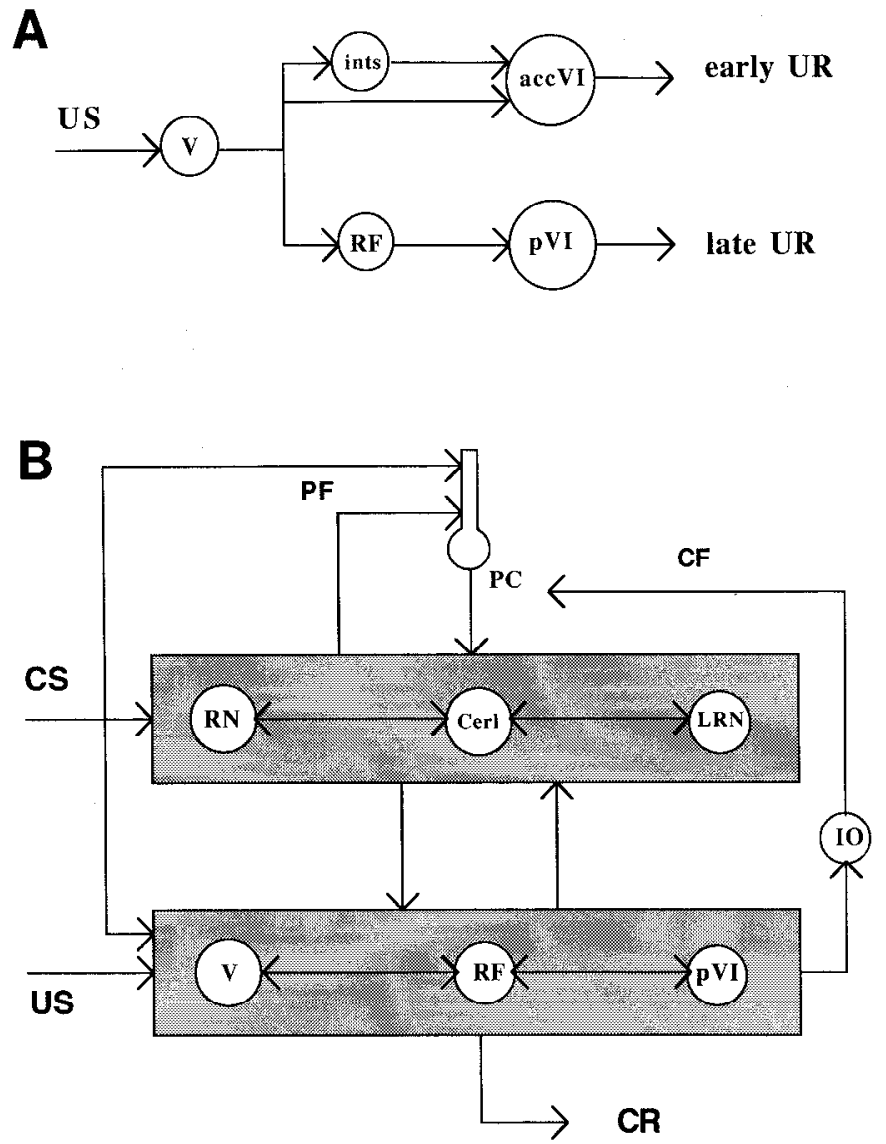

Figure 10. Schematic diagram of the postulated UR and CR pathways. $A$, The UR pathway. The trigeminal nerve US activates both the principal $(p V I)$ and accessory $(a c c V I)$ abducens motor nuclei via the trigeminal nucleus $(V)$ to produce the UR. The accessory nucleus produces an early short-duration component of the UR either through interneurons (ints) or by direct activation. The principal nucleus produces a late long-duration component of the UR which is generated through the caudal reticular formation possibly by a positive feedback circuit. $B$, The CR pathway includes that portion of the UR pathway that generates the late response, namely, nucleus V, RF and pVI. For simplicity, we have called this the reticuloabducens network and draw a box around it to indicate that it is a positive feedback circuit. In addition, the $\mathrm{CR}$ pathway includes the cerebellorubral network, boxed in above, which is a recurrent circuit consisting of interconnections between RN, Cerl and LRN. Both networks communicate with one another and both networks receive information about the US and the CS. The Discussion describes how the cerebellorubral network interfaces with the reticuloabducens network to produce CRs, and mechanisms whereby the reticuloabducens network may generate CRs in the absence of the cerebellum. See other figure captions for abbreviations, except $I O$, inferior olive; $C F$, climbing fiber; $P F$, parallel fiber.

single-unit recording studies support a role for the trigeminal nucleus and reticular formation in producing CRs (Desmond and Moore, 1986; Richards et al., 1991). Response latencies of CRrelated activity in these areas have a wide range, from about 110 msec before the CR to $46 \mathrm{msec}$ after it. Such wide ranging latencies may reflect positive feedback in a circuit linking the trigeminal nucleus with the reticular formation, and with the cerebellum and red nucleus, as we have proposed in Figure $10 B$. The following interpretation addresses both the origin of activity in the cerebellorubral circuit and the manner in which it interfaces with the reticuloabducens network to generate CRs. We also discuss mechanisms whereby CRs may potentially be generated in the absence of the cerebellorubral circuitry. 
The cerebellorubral network is organized in a modular fashion such that individual loops transmit motor commands to a distinct combination of muscles and are controlled by a particular strip of Purkinje cells (Houk et al., 1990, 1993). The initiation of conditioned activity in appropriate loops would require a CS input to the cerebellorubral network to initiate positive feedback coupled with a silencing of the Purkinje cells (PC) that inhibit the loop so as to release the feedback. Once initiated, activity in the cerebellorubral loops might require some time to spread to the reticuloabducens network, and this might explain the lead time between cerebellorubral bursts and CRs. The postulated pause in PC discharge would not explain why PCs were labeled by sulforhodamine. Instead, the labeled PCs might be related to increased PC activity associated with CR extinction that occurred during the period of exposure to sulforhodamine. While there is considerable evidence for a cerebellar role in conditioning, there is also evidence that some low-level conditioning can be generated in preparations lacking a cerebellum (Norman et al., 1977; Kelly et al., 1990; Yeo, 1991; Harvey et al., 1993; Keifer, 1993b; also Gruart et al., 1994). Since the reticuloabducens network receives information about both the CS and US, it is possible that some low-level conditioning occurs in this pathway, which would explain the findings cited above. The potential availability of this simple mechanism raises the question as to the function of the more elaborate cerebellar mechanism. One important feature of the cerebellar circuit is that it provides not only a mechanism for appropriate timing of CRs (Perrett et al., 1993), but also a mechanism for adjusting their amplitude and duration (Houk, 1990).

\section{References}

Andrews O (1915) The ability of turtles to discriminate sounds. Bull Wisc Nat Hist Soc 13:189-195.

Art JJ, Fettiplace R (1984) Efferent desensitization of auditory nerve fibre responses in the cochlea of the turtle Pseudemys scripta elegans. J Physiol (Lond) 356:507-523.

Barbas-Henry HA, Lohman AHM (1986) The motor complex and primary projections of the trigeminal nerve in the monitor lizard, Varanus exanthematicus. J Comp Neurol 254:314-329.

Barbas-Henry HA, Lohman AHM (1988) The motor nuclei and sensory neurons of the IIIrd, IVth, and VIth cranial nerves in the monitor lizard, Varanus exanthematicus. J Comp Neurol 267:370-386.

Barbas-Henry HA, Wouterlood FG (1988) Synaptic connections between primary trigeminal afferents and accessory abducens motoneurons in the monitor lizard, Varanus exanthematicus. J Comp Neurol 267:387-397.

Berthier NE, Moore JW (1986) Cerebellar Purkinje cell activity related to the classically conditioned nictitating membrane response. Exp Brain Res 63:341-350.

Berthier NE, Moore JW (1990) Activity of deep cerebellar nuclear cells during classical conditioning of nictitating membrane extension in rabbits. Exp Brain Res 83:44-54.

Bloedel JR, Bracha V, Kelly TM, Wu J-Z (1991) Substrates for motor learning: does the cerebellum do it all? Ann NY Acad Sci 627:305318.

Boycott BB, Guillery RW (1962) Olfactory and visual learning in the red-eared terrapin, Pscudemys scripta elegans. J Exp Biol 39:567577.

Cassella JV, Harty TP, Davis M (1986) Fear conditioning, pre-pulse inhibition and drug modulation of a short latency startle response measured electromyographically from neck muscles in the rat. Physiol Behav 36:1187-1191.

Cegavske CF, Patterson MM, Thompson RF (1979) Neuronal unit activity in the abducens nucleus during classical conditioning of the nictitating membrane response in the rabbit (Oryctolagus cuniculus). J Comp Physiol Psychol 93:595-609.

Chang, JJ, Gelperin A (1980) Rapid taste-aversion learning by an isolated molluscan central nervous system. Proc Natl Acad Sci USA 77: 6204-6206.
Davis M (1984) The mammalian startle response. In: Neural mechanisms of startle behavior (Eaton RC, ed), pp 287-351. New York: Plenum.

Delgado-Garcia JM, Evinger C, Escudero M, Baker R (1990) Behavior of accessory abducens motoneurons during eye retraction and rolation in the alert cat. J Neurophysiol 64:413-422.

Desmond JE, Moore JW (1986) Dorsolateral pontine tegmentum and the classically conditioned nictitating membrane response: analysis of CR-related single-unit activity. Exp Brain Res 65:59-74.

Desmond JE, Moore JW (1991) Single-unit activity in red nucleus during the classically conditioned rabbit nictitating membrane response. Neurosci Res 10:260-279.

Disterhoft JF, Quinn KJ, Weiss C, Shipley MT (1985) Accessory abducens nucleus and conditioned eye retraction/nictitating membrane extension in rabbit. J Neurosci 5:941-950.

Donegan NH (1981) Priming-produced facilitation or diminution of responding to a Pavlovian unconditioned stimulus. J Exp Psychol 7:295-312.

Evinger C, Manning KA (1993) Pattern of extraocular muscle activation during reflex blinking. Exp Brain Res 92:502-506.

Farley J, Alkon DL (1987) In vitro associative conditioning of Hermissenda: cumulative depolarization of type B photoreceptors and short-term associative behavioral changes. J Neurophysiol 57:16391668.

Foster RE, Hall WC (1978) The organization of central auditory pathways in a reptile, Iguana iguana. J Comp Neurol 178:783-832.

Glickstein M, Yeo C (1990) The cerebellum and motor learning. J Cognit Neurosci 2:69-80.

Gormezano I (1972) Investigations of defense and reward conditioning in the rabbit. In: Classical conditioning. II. Current research and theory (Black AH, Prokasy WF, eds). New York: Appleton.

Gormezano I, Schneiderman N, Deaux E, Fuentes I (1962) Nictitating membrane: classical conditioning and extinction in the albino rabbit. Science 138:33-34.

Gormezano I, Kehoe EJ, Marshall BS (1983) Twenty ycars of classical conditioning research with the rabbit. Prog Psychobiol Physiol Psychol 10:197-275.

Graf V, Tighe T (1971) Subproblem analysis of discrimination shift learning in the turtle (Chrysemys picta picta). Psychon Sci 25:257259.

Gruart A, Blazquez P, Delgado-Garcia JM (1994) Kinematic analysis of classically-conditioned eyelid movements in the cat suggest a brain stem site for motor learning. Neurosci Lett 175:81-84.

Harvey JA, Welsh JP, Yeo CH, Romano AG (1993) Recoverable and nonrecoverable deficits in conditioned responses after cerebellar cortical lesions. J Neurosci 13:1624-1635.

Houk JC (1990) Role of cerebellum in classical conditioning. Soc Neurosci Abstr 16:474

Houk JC, Singh SP, Fisher C, Barto AG (1990) An adaptive sensorimotor network inspired by the anatomy and physiology of the cerebellum. In: Neural networks for control (Miller WT, Sutton RS, Werbos PJ, eds), pp 301-348. Cambridge, MA: MIT Press.

Houk JC, Keifer J, Barto AG (1993) Distributed motor commands in the limb premotor network. Trends Neurosci 16:27-33.

Keifer J (1993a) In vitro eye-blink reflex model: role of excitatory amino acids and labeling of network activity with sulforhodamine. Exp Brain Res 97:239-253.

Keifer J (1993b) The cerebellum and red nucleus are not required for classical conditioning of an in vitro model of the eye-blink reffex. Soc Neurosci Abstr 19:1001.

Keifer J, Houk JC (1990) Conditioned response learning in the in vitro turtle brainstem-cerebellum. Soc Neurosci Abstr 16:763.

Keifer J, Houk JC (1991) Role of excitatory amino acids in mediating burst discharge of red nucleus neurons in the in vitro turtle brainstem-cerebellum. J Neurophysiol 65:454-467.

Keifer J, Houk JC (1994) Motor function of the cerebellorubrospinal system. Physiol Rev 74:509-542.

Keifer J, Vyas D, Houk JC (1992) Sulforhodanine labeling of neural circuits engaged in motor pattern generation in the in vitro turtle brainstem-cerebellum. J Neurosci 12:3187-3199.

Kelly TM, Zuo C-C, Bloedel JR (1990) Classical conditioning of the eyeblink reflex in the decerebrate--decerebellate rabbit. Behav Brain Res 38:7-18.

Kjaerulff O, Barajon I, Kiehn O (1994) Sulforhodamine-labelled cells 
in the neonatal rat spinal cord following chemically induced locomolor aclivily in vitro. J Physiol (Lond) 478:265-273.

Leaton RN, Supple WF (1986) Cerebellar vermis: essential for longterm habituation of the acoustic startle response. Science 232:513515.

Lukowiak K, Sahley C (1981) The in vitro classical conditioning of the gill withdrawal reflex of Aplysia californica. Science 212:15161518.

Manton ML, Karr A, Ehrenfeld DW (1972) An operant method for the study of chemoreception in the green turtle, Chelonia mydas. Brain Behav Evol 5:188-201.

Marcus LL (1981) Veterinary biology and medicine of captive amphibians and reptiles. Philadelphia: Lea and Febiger.

Mauk MD, Thompson RF (1987) Retention of classically conditioned eyelid responses following acute decerebration. Brain Res 403:8995.

McCormick DA, Thompson RF (1984) Neuronal responses of the rabbit cerebellum during acquisition and performance of a classically conditioned nictitating membrane-eyelid response. J Neurosci 4:2811-2822.

Moyer JR, Deyo RA, Disterhoft JF (1990) Hippocampectomy disrupts trace eye-blink conditioning in rabbits. Behav Neurosci 104:243-252.

Mpitsos GJ, Davis WJ (1973) Learning: classical and avoidance conditioning in the mollusk Pleurobranchaea. Science 180:317-320.

Norman RJ, Buchwald JS, Villablanca JR (1977) Classical conditioning with auditory discrimination of the eye blink in decerebrate cats. Science 196:551-553.

Patterson WC (1966) Hearing in the turtle. J Aud Res 6:453-464.

Perrett SP, Ruiz BP, Mauk MD (1993) Cerebellar cortex lesions disrupt learning-dependent timing of conditioned eyelid responses. J Neurosci 13:1708-1718.

Pert A, Bitterman ME (1969) A technique for the study of consummatory behavior and instrumental learning in the turtle. Am Psychol 24:258-261.

Pilz PKD, Caeser M, Ostwald J (1988) Comparative threshold studies of the acoustic pinna, jaw and stapes reflex in the cat. Physiol Behav 43:411-415.
Poliakov KL (1930) The physiology of the olfactory and auditory analyzers in the turtle. Russian J Gen Physiol 13:161-178.

Prosser CL, Hunter WS (1936) The extinction of startle responses and spinal reflexes in the white rat. Am J Physiol 117:609-618.

Richards WG, Ricciardi TN, Moore JW (1991) Activity of spinal trigeminal pars oralis and adjacent reticular formation units during differential conditioning of the rabbit nictitating membrane response. Behav Brain Res 44:195-204.

Sarrafizadeh R, Houk JC (1994) Anatomical organization of the limb premotor network in the turtle (Chrysemys picta) revealed by in vitro transport of biocytin and neurobiotin. J Comp Neurol 344:137-158.

Schneiderman N, Gormezano I (1964) Conditioning of the nictitating membrane of the rabbit as a function of CS-US interval. J Comp Physiol Psychol 57:188-195.

Sharp FR, Gonzalez MF, Sharp JW, Sagar SM (1989) c-fos expression and (14C) 2-deoxyglucose uptake in the caudal cerebellum of the rat during motor/sensory cortex stimulation. J Comp Neurol 284:621636.

Solomon PR, Vander Schaaf ER, Weisz DJ, Thompson RF (1986) Hippocampus and trace conditioning of the rabbit's classically conditioned nictitating membrane response. Behav Neurosci 5:729-744.

Spigel IM (1963) Running speed and intermediate brightness discrimination in the fresh water turtle (Chrysemys). J Comp Physiol Psychol 56:924-928.

Steinmetz JE, Lavond DG, Ivkovich D, Logan CG, Thompson RF (1992) Disruption of classical eyelid conditioning after cerebellar lesions: damage to a memory trace system or a simple performance deficit? J Neurosci 12:4403-4426.

Ten Donkelaar HJ, Nieuwenhuys R (1979) The brainstem. In: Biology of the reptila, Vol 10 (Gans C, ed), pp 133-200. New York: Academic.

Thompson RF (1986) The neurobiology of learning and memory. Science 233:941-947.

Underwood G (1970) The eye. In: Biology of the reptilia, Vol 2 (Gans C, ed), pp 1-97. New York: Academic.

Yeo CH (1991) Cerebellum and classical conditioning of motor responses. Ann NY Acad Sci 627:292-304.

Yeo CH, Hardiman MJ, Glickstein M (1985) Classical conditioning of the nictitating membrane response of the rabbit. I. Lesions of the cerebellar nuclei. Exp Brain Res 60:87-98. 